\title{
DEPRESSION OF PHAGOCYTOSIS BY SOLUTES IN CONCENTRATIONS FOUND IN THE KIDNEY AND URINE*
}

\author{
BY IRWIN CHERNEW AND A. I. BRAUDE \\ (From the Department of Medicine, University of Pittsburgh School of Medicine, \\ Pittsburgh, Pa.)
}

(Submitted for publication May 18, 1962; accepted July 5, 1962)

Immunologic processes in the kidney encounter wide variations in solute concentration and osmotic pressure that exist in no other extracellular fluids (1). It can be anticipated, for example, that leukocytes in the kidney might be exposed to $425 \mathrm{mM}$ per $\mathrm{L}$ sodium and $850 \mathrm{mM}$ per L urea, tissue concentrations demonstrated in the renal papillae of mammals. Between the extremes of maximum diuresis and antidiuresis, phagocytic cells might be exposed to tubular fluid that ranges from under 50 to over $1,300 \mathrm{mOsm}$ per L, or approximately one-sixth to four times the osmotic pressure of plasma (1). In order to determine how leukocytes function in this environment, the present study was made of phagocytosis in urine and in improvised fluids containing solutes in the concentrations found in the kidney and urinary tract. The results show that such concentrations of urinary solutes depress phagocytosis and may thereby contribute to the unique susceptibility of the kidney to infection by bacteria that seldom produce disease elsewhere.

\section{METHODS}

\section{A. Technique for studying phagocytosis}

Four-tenths (0.4) $\mathrm{ml}$ fresh heparinized human venous blood or $0.2 \mathrm{ml}$ plasma-leukocyte suspensions were added to $2 \mathrm{ml}$ of test solution and mixed with $0.2 \mathrm{ml}$ of bacterial suspensions in sterile pyrogen-free glass tubes 10 to $11 \mathrm{~mm}$ in diameter and $10 \mathrm{~cm}$ long. The tubes were immediately stoppered and rotated mechanically at 5 revolutions per minute at $37^{\circ} \mathrm{C}$. Tubes containing staphylococci were rotated for 30 minutes and those containing Escherichia coli for 2 hours, periods found in preliminary studies to be required for heavy phagocytosis of either species of bacteria. After rotation the tubes were inverted 10 to 15 times, and smears prepared on glass slides for staining with combined Wright-Giemsa's stain.

The number of bacteria per 100 neutrophiles and the per cent of cells exhibiting phagocytosis were compared

* Supported in part by grant H-3220, U. S. Public Health Service. with control values. The results are indicated in the tables by the following symbols: $\% \mathrm{P}=$ per cent neutrophiles exhibiting phagocytosis; $\mathrm{B} / \mathrm{N}_{\mathbf{x}}=$ number bacteria ingested per 100 neutrophiles in test solution; $B / N_{c}=$ number bacteria ingested per 100 neutrophiles in control solution; \%C, phagocytic index, $=\mathrm{B} / \mathrm{N}_{\mathrm{x}} \div \mathrm{B} / \mathrm{N}_{\mathrm{c}} \times 100$; a subscript indicates the number of neutrophiles containing too many bacteria to count, e.g., $\mathrm{B} / \mathrm{N}_{\mathrm{x}}=2500_{-5}$ indicates that 2,250 bacteria were counted in 95 neutrophiles and 5 neutrophiles contained too many bacteria to count. For purposes of calculating $\% \mathrm{C}$, it was arbitrarily assumed that such phagocytic cells contained 50 bacteria each.

\section{B. Preparation of plasma-leukocyte suspensions}

The red cells in heparinized venous human blood were allowed to become sediment at $37^{\circ} \mathrm{C}$ and the plasma-leukocyte supernatant fluid was removed for study. The leukocyte count was 1.5 to 2.0 times that of the original whole blood. Plasma-leukocyte suspensions were used as indicated below; otherwise the experiments were done with fresh heparinized whole blood to avoid the injury to leukocytes that might occur during separation from red cells. The presence of plasma constituents and erythrocytes in the test suspensions was considered desirable from the standpoint of reproducing conditions of phagocytosis that exist in the kidney and urine during phagocytosis.

\section{Preparation of bacterial suspensions}

1. Staphylococci. A strain of Staphylococcus epidermidis isolated from infected human urine was grown in trypticase soy broth for 18 to 20 hours at $37^{\circ} \mathrm{C}$ and then killed by heating for 30 minutes at $72^{\circ} \mathrm{C}$. The bacterial count of this suspension was $2.0 \times 10^{6}$ per $\mathrm{mm}^{3}$ as determined in a hemocytometer chamber. The dead nonpathogenic staphylococci were used because they permitted a study of phagocytosis of particles offering no discernible resistance to ingestion.

2. Escherichia coli. A strain of E. coli (Serotype $0: 113$ ) isolated from infected human urine was cultured in trypticase soy broth for approximately 18 hours and the bacterial growth resuspended in Ringer's solution. A fresh culture of $E$. coli was prepared for each experiment and the concentration of bacilli determined with the hemocytometer. Most suspensions contained about $10^{6}$ bacteria per $\mathrm{mm}^{3}$ with a range from $3.6 \times 10^{5}$ per $\mathrm{mm}^{3}$ to 1.8 $\times 10^{6}$ per $\mathrm{mm}^{3}$. After mixture with blood or plasmaleukocytes, the concentration of bacteria was about 7 to 8 per cent of the original. The ratios of bacteria to 
leukocytes ranged from $70: 1$ to $400: 1$ and were kept constant in each experiment. These ratios of $E$. coli to leukocytes were selected in order to reproduce the rigorous challenge to phagocytosis encountered in heavily infected pyuric urines. The high consistency of the results recorded below were not improved when extracellular bacteria were removed by washing the leukocytes in lactated Ringer's solution just before preparing the smears.

\section{Preparation of test solutions}

Lactated Ringer's solution was used as the basic medium for phagocytosis and the solutes were altered according to the aims of the experiments; cation concentrations were adjusted by changing the concentration of their chloride salts. Ringer-Locke solution was used for titrating the effect of variable sodium concentrations on phagocytosis. The formula of the lactated Ringer's solution (Abbott) was $130 \mathrm{mM}$ per $\mathrm{L} \mathrm{Na}, 4 \mathrm{mM}$ per $\mathrm{L} \mathrm{K}$, $1.5 \mathrm{mM}$ per $\mathrm{L} \mathrm{Ca}, 109 \mathrm{mM}$ per $\mathrm{L} \mathrm{Cl}$, and $28 \mathrm{mM}$ per $\mathrm{L}$ lactate. Ringer-Locke solution was prepared by adding the desired quantities of solute to distilled water; it contained 130 to $150 \mathrm{mM}$ per $\mathrm{L} \mathrm{NaCl}, 3$ to $5 \mathrm{mM}$ per $\mathrm{L}$ $\mathrm{KCl}, 1.5 \mathrm{mM}$ per $\mathrm{L} \mathrm{CaCl}_{2}, 100$ to $200 \mathrm{mg}$ per cent glucose, and $4.0 \mathrm{mM}$ per $\mathrm{L} \mathrm{NaHCO}_{3}$. The exact concentrations of solutes are recorded for individual experiments. ${ }^{1}$

\section{E. Preparation of urine}

Most studies were conducted with whole fresh urines, or with the cell-free supernatant fluids of freshly obtained pyuric urines. A few urines were stored at $-20^{\circ} \mathrm{C}$ before the experiment.

Osmolarities of the urines were measured in the Fiske osmometer and $\mathrm{pH}$ of the final mixtures in the Beckman $\mathrm{pH}$ meter. Sodium and potassium were determined by photometry, urea by urease nesslerization, and chloride by titration with mercuric ion. The relative degree of phagocytosis in urine was based on control values determined simultaneously in lactated Ringer's solution.

\section{F. Adjustment of $p H$}

The effect of $\mathrm{pH}$ on phagocytosis was studied after diluting $0.8 \mathrm{ml}$ of a plasma-leukocyte suspension with 4.0 ml lactated Ringer's solution; then $0.06 \mathrm{~N} \mathrm{HCl}$ was

\footnotetext{
1 The final concentration of solute depends not only on the concentration in the original test solution, the plasma, and the bacterial suspension, but also on the amount of solute diffusing into the cells. Only the concentrations in the original solution are listed. The final concentration of solutes, such as urea, that are present in lower concentration in the blood than in the test solution would be about 25 per cent less than the recorded value. In the solutions studied for the effects of high sodium concentrations, the actual concentrations of this solute would be 1 to 10 per cent less after blood and bacteria were added. In test solutions with low sodium values, the final difference was not greater than 10 per cent except for the lowest concentration, which doubled in the final solution.
}

added until the desired $\mathrm{pH}$ was obtained as determined in the Beckman $\mathrm{pH}$ meter. Two $\mathrm{ml}$ of each mixture was removed to determine $\mathrm{pH}$; the remaining $2.8 \mathrm{ml}$ was inoculated with $0.2 \mathrm{ml}$ of the standard $E$. coli suspension and phagocytosis determined in the usual fashion. The white blood cell (WBC) count of the plasma-leukocyte suspension before dilution with lactated Ringer's solution was approximately twice that of blood, and its erythrocyte count, approximately $10^{4}$ per $\mathrm{mm}^{3}$. The addition of each $0.1 \mathrm{ml}$ of $0.06 \mathrm{~N} \mathrm{HCl}$ raised chloride concentration approximately 3 to $4 \mathrm{mM}$ per L.

\section{RESULTS}

\section{Phagocytosis in improvised fluids}

A. Sodium. As shown in Table I, sodium in concentrations of $300 \mathrm{mM}$ per $\mathrm{L}$ and greater markedly reduced phagocytosis of both staphylococci and E. coli. At concentrations of sodium below $300 \mathrm{mM}$ per L, hypertonic solutions produced little depression of phagocytosis. Phagocytosis also appeared to be reduced when the concentration of sodium fell below $100 \mathrm{mM}$ per $\mathrm{L}$.

Disturbed phagocytosis in high and low sodium concentration was associated with morphologic abnormalities. The cells in solutions of high sodium concentrations were shrunken, and their nuclei were often fused or in the process of being extruded. Clumping of cells, observed in the control solutions, also failed to occur. In the dilute solutions, the neutrophiles were sometimes swollen and clumped.

B. Urea. As shown in Tables II and III, phagocytosis was depressed at least 50 per cent at urea concentrations above $500 \mathrm{mM}$ per L, and impairment became progressively reduced until marked inhibition was observed at $1,000 \mathrm{mM}$ per L. The cells were usually reduced in size in the highly concentrated solution. The nuclear lobes of some neutrophiles had fused.

C. Sodium and urea in combination. In order to determine if the deleterious effects of high concentrations of sodium and urea were additive, both were studied in the same solution. The results are given in Table IV.

Addition of urea to solutions already hypertonic with $250 \mathrm{mM}$ per L sodium further depressed phagocytosis of $E$. coli, and to a much greater degree than when those concentrations of urea were added to solutions isotonic with 150 $\mathrm{mM}$ per L sodium. In hypotonic solutions of sodium, the addition of urea also impaired phago- 
TABLE I

Phagocytosis in varyıng concentrations of sodium chloride*

\begin{tabular}{|c|c|c|c|c|c|c|c|c|c|c|}
\hline & & \multicolumn{9}{|c|}{$\mathrm{m} \mathbf{M} / \mathrm{L}$ Sodium } \\
\hline & & 392 & \multicolumn{2}{|l|}{324} & 244 & 167 & \multicolumn{2}{|l|}{$157 \dagger$} & 92 & 11.3 \\
\hline \multicolumn{11}{|c|}{ Phagocytosis of Staphylococci } \\
\hline $\begin{array}{l}\text { Subject } 1 \text {, } \\
\text { whole blood }\end{array}$ & $\begin{array}{l}\% \mathrm{P} \\
\mathrm{B} / \mathrm{N}_{x} \\
\% \mathrm{C}\end{array}$ & $\begin{array}{r}37 \\
166 \\
6.3\end{array}$ & $\begin{array}{c}64 \\
296 \\
11.3\end{array}$ & & $\begin{array}{c}100 \\
1567_{-4}^{-4} \\
59.8\end{array}$ & $\begin{array}{l}100^{1} \\
2810_{-8} \\
100^{-8}\end{array}$ & $\begin{array}{l}100^{16} \\
2620_{-6} \\
100^{-6}\end{array}$ & & $\begin{array}{r}100 \\
2414_{-3} \\
92.1\end{array}$ & $\begin{array}{c}59 \\
981_{-3} \\
43.2\end{array}$ \\
\hline \multirow[t]{2}{*}{$\begin{array}{l}\text { Subject } 2 \text {, } \\
\text { whole blood }\end{array}$} & \multirow[t]{2}{*}{$\begin{array}{l}\% \mathrm{P} \\
\mathrm{B} / \mathrm{N}_{\mathrm{x}} \\
\% \mathrm{C}\end{array}$} & $\begin{array}{c}29 \\
104 \\
10.2\end{array}$ & $\begin{array}{c}32 \\
159 \\
15.6\end{array}$ & \multicolumn{2}{|r|}{$\begin{array}{c}64 \\
931_{-5} \\
91_{1.3}\end{array}$} & $\begin{array}{r}90 \\
867_{-3} \\
94.8\end{array}$ & \multicolumn{2}{|l|}{$\begin{array}{l}81 \\
1020_{-6} \\
100^{-}\end{array}$} & $\begin{array}{c}78 \\
926 \\
90.8\end{array}$ & $\begin{array}{r}26 \\
225 \\
22.1\end{array}$ \\
\hline & & 400 & 350 & 300 & 250 & 200 & $159 \dagger$ & 100 & 50 & 25 \\
\hline \multicolumn{11}{|c|}{ Phagocytosis of $E$. coli } \\
\hline $\begin{array}{l}\text { Subject } 3 \text {, } \\
\text { whole blood }\end{array}$ & $\begin{array}{l}\% \mathrm{P} \\
\mathrm{B} / \mathrm{N}_{\mathbf{x}} \\
\%_{0} \mathrm{C}\end{array}$ & $\begin{array}{l}32 \\
36 \\
4.1\end{array}$ & & $\begin{array}{c}47 \\
237 \\
27.2\end{array}$ & & $\begin{array}{c}91 \\
699 \\
80.2\end{array}$ & $\begin{array}{r}87 \\
871 \\
100\end{array}$ & $\begin{array}{c}89 \\
694 \\
79.7\end{array}$ & $\begin{array}{c}71 \\
423 \\
48.6\end{array}$ & $\begin{array}{c}72 \\
308 \\
35.4\end{array}$ \\
\hline $\begin{array}{l}\text { Plasma- } \\
\text { leukocyte } \\
\text { suspension }\end{array}$ & $\begin{array}{l}\% \mathrm{P} \\
\mathrm{B} / \mathrm{N}_{\mathrm{x}} \\
\% \mathrm{C}\end{array}$ & $\begin{array}{l}1 \\
2 \\
7\end{array}$ & $\begin{array}{l}12 \\
48 \\
16.6\end{array}$ & $\begin{array}{l}15 \\
68 \\
23.5\end{array}$ & $\begin{array}{l}20 \\
78 \\
27\end{array}$ & $\begin{array}{r}37 \\
183 \\
63.3\end{array}$ & $\begin{array}{r}82 \\
289 \\
100\end{array}$ & $\begin{array}{c}94 \\
387 \\
133.4\end{array}$ & & \\
\hline
\end{tabular}

$* \% \mathrm{P}=$ per cent neutrophiles exhibiting phagocytosis; $\mathrm{B} / \mathrm{N}_{\mathrm{x}}=$ number bacteria ingested per 100 neutrophiles in test solution; $\mathrm{B} / \mathrm{N}_{\mathrm{c}}=$ number bacteria ingested per 100 neutrophiles in control solution; $\% \mathrm{C}$, phagocytic index, $=\mathrm{B} / \mathrm{N}_{\mathrm{x}}$ $\div \mathrm{B} / \mathrm{N}_{\mathrm{c}} \times 100$; a subscript indicates the number of neutrophiles containing too many bacteria to count. See Methods, section $A$, for details.

$\dagger$ This is the control solution. The basic solution is a Ringer-Locke solution varied only in sodium concentration.

cytosis, but less than in hypertonic solution of sodium.

The observation that phagocytosis in $250 \mathrm{mM}$ per L sodium was only slightly reduced but became markedly depressed with the addition of 120 $\mathrm{mM}$ per $\mathrm{L}$ urea might indicate that an osmotic pressure critical to inhibition of phagocytosis had been reached. A concentration of $324 \mathrm{mM}$ per $\mathrm{L}$ sodium chloride sharply inhibited phagocytosis, while $244 \mathrm{mM}$ per L sodium chloride was only moderately inhibitory (see Table I). The addition of $120 \mathrm{mM}$ per $\mathrm{L}$ urea is equivalent to about $60 \mathrm{mM}$ per $\mathrm{L}$ sodium chloride in osmolarity.
Thus, osmolarity contributed by $324 \mathrm{mM}$ per L sodium chloride would be approximately equivalent to that contributed by $250 \mathrm{mM}$ per $\mathrm{L}$ sodium chloride and $120 \mathrm{mM}$ per L urea.

In high concentrations of urea and sodium, the cells were usually small; in low sodium and 0 $\mathrm{mM}$ per $\mathrm{L}$ urea, cells were swollen, vacuolated, and clumped.

D. Potassium, calcium, creatinine, ammonium, and citrate. The effects on phagocytosis of each solute was determined when its concentration was manipulated in Ringer-Locke solution to represent values near or beyond the extreme that might

TABLE II

Phagocytosis of Staphylococci in varying concentrations of urea*

\begin{tabular}{|c|c|c|c|c|c|c|c|}
\hline & & \multicolumn{6}{|c|}{$\mathrm{mM} / \mathrm{L}$ Urea $\dagger$} \\
\hline & & of & 102 & 360 & 600 & 900 & 1,200 \\
\hline \multirow{2}{*}{$\begin{array}{c}\text { Subject } 3 \text {, } \\
\text { whole } \\
\text { blood }\end{array}$} & $\begin{array}{l}\% \mathrm{P} \\
\mathrm{B} / \mathrm{N}_{\mathrm{x}}\end{array}$ & $\begin{array}{c}100 \\
3,083_{-3}\end{array}$ & $\begin{array}{c}99 \\
2,187_{-4}\end{array}$ & $\begin{array}{r}96 \\
1,704\end{array}$ & $\begin{array}{r}61 \\
477\end{array}$ & $\begin{array}{r}41 \\
440\end{array}$ & $\begin{array}{r}44 \\
292\end{array}$ \\
\hline & $\% \mathrm{C}$ & 100 & 70.9 & 55.3 & 15.3 & 14.3 & 9.5 \\
\hline
\end{tabular}

* Symbols as in Table I.

$\dagger$ Because of dilution by blood, the final concentration is approximately 25 per cent less than that recorded here in the test solution (see Methods, section D). The final concentration after addition of test solution with $900 \mathrm{mM}$ per $\mathrm{L}$ urea is approximately $675 \mathrm{mM}$ per L, a figure well below that in the dog renal medulla (1).

$\ddagger$ Control solution. 
TABLE III

Phagocytosis of E. coli in varying concentrations of urea*

\begin{tabular}{|c|c|c|c|c|c|c|c|c|c|c|c|c|}
\hline & & \multicolumn{11}{|c|}{$\mathrm{m} \mathbf{M} / \mathbf{L}$ Urea } \\
\hline & & of & 100 & 200 & 300 & 400 & 500 & 600 & 700 & 800 & 900 & 1,000 \\
\hline $\begin{array}{l}\text { Subject } 4 \text {, } \\
\quad \text { whole blood } \ddagger\end{array}$ & $\begin{array}{l}\% \mathrm{P} \\
\mathrm{B} / \mathrm{N}_{\mathbf{x}} \\
\% \mathrm{C}\end{array}$ & $\begin{array}{r}97 \\
100 \\
100\end{array}$ & $\begin{array}{c}80 \\
511 \\
71.8\end{array}$ & & 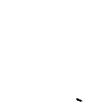 & & $\begin{array}{c}61 \\
324 \\
45.5\end{array}$ & $\begin{array}{c}62 \\
348 \\
48.9\end{array}$ & & & & \\
\hline $\begin{array}{l}\text { Subject } 5 \text {, } \\
\quad \text { whole blood } \ddagger\end{array}$ & $\begin{array}{l}\% \mathrm{P} \\
\mathrm{B} / \mathrm{N}_{x} \\
\% \mathrm{C}\end{array}$ & $\begin{array}{r}90 \\
429 \\
100\end{array}$ & $\begin{array}{c}87 \\
359 \\
83.7\end{array}$ & & & & $\begin{array}{c}69 \\
250 \\
58.3\end{array}$ & $\begin{array}{c}51 \\
107 \\
25.0\end{array}$ & & & & \\
\hline $\begin{array}{l}\text { Subject } 6 \text {, } \\
\quad \text { whole blood } \ddagger\end{array}$ & $\begin{array}{l}\% \mathrm{P} \\
\mathrm{B} / \mathrm{N}_{\mathrm{x}} \\
\% \mathrm{C}\end{array}$ & $\begin{array}{r}98 \\
749 \\
100\end{array}$ & $\begin{array}{r}98 \\
518 \\
59\end{array}$ & $\begin{array}{r}97 \\
636 \\
85\end{array}$ & $\begin{array}{r}90 \\
431 \\
58\end{array}$ & $\begin{array}{r}95 \\
519 \\
69\end{array}$ & $\begin{array}{r}85 \\
329 \\
44\end{array}$ & $\begin{array}{r}72 \\
213 \\
28\end{array}$ & $\begin{array}{r}51 \\
187 \\
25\end{array}$ & $\begin{array}{r}25 \\
150 \\
20\end{array}$ & $\begin{array}{l}33 \\
88 \\
12\end{array}$ & $\begin{array}{r}11 \\
44 \\
6\end{array}$ \\
\hline $\begin{array}{l}\text { Subject } 7, \\
\text { plasma- } \\
\text { leukocyte } \\
\text { suspension } \ddagger\end{array}$ & $\begin{array}{l}\% \mathrm{P} \\
\mathrm{B} / \mathrm{N}_{\mathrm{x}} \\
\% \mathrm{C}\end{array}$ & $\begin{array}{r}99 \\
452 \\
100\end{array}$ & & & $\begin{array}{c}87 \\
355 \\
78.5\end{array}$ & $\begin{array}{r}69 \\
257 \\
56.9\end{array}$ & $\begin{array}{c}81 \\
356 \\
78.8\end{array}$ & $\begin{array}{c}33 \\
143 \\
31.6\end{array}$ & & & & $\begin{array}{l}4 \\
9 \\
2.0\end{array}$ \\
\hline
\end{tabular}

* Symbols as in Table I.

$\dagger$ Control solution.

‡ Suspended in lactated Ringer's solution as described in Methods, section A.

TABLE IV

Combined effect of sodium and urea on phagocytosis of E. coli in Ringer-Locke solution*

\begin{tabular}{|c|c|c|c|c|c|c|c|c|c|c|c|c|}
\hline \multicolumn{2}{|c|}{$\begin{array}{c}\mathrm{mM} / \mathrm{L} \text { Sodium } \\
\mathrm{mM} / \mathrm{L} \text { Urea }\end{array}$} & $\begin{array}{r}250 \\
0\end{array}$ & $\begin{array}{l}250 \\
120\end{array}$ & $\begin{array}{l}250 \\
400\end{array}$ & $\begin{array}{r}150 \\
0\end{array}$ & $\begin{array}{l}150 \\
120\end{array}$ & $\begin{array}{l}150 \\
400\end{array}$ & $\begin{array}{l}150 \\
600\end{array}$ & $\begin{array}{r}50 \\
0\end{array}$ & $\begin{array}{r}50 \\
120\end{array}$ & $\begin{array}{r}50 \\
400\end{array}$ & $\begin{array}{r}50 \\
600\end{array}$ \\
\hline Subject 7 & $\begin{array}{l}\% \mathrm{P} \\
\mathrm{B} / \mathrm{N}_{\mathrm{x}} \\
\% \mathrm{C}\end{array}$ & $\begin{array}{c}63 \\
218 \\
50.8\end{array}$ & $\begin{array}{l}27 \\
59 \\
13.8\end{array}$ & $\begin{array}{c}19 \\
34 \\
7.9\end{array}$ & $\begin{array}{r}90 \\
429 \\
100\end{array}$ & $\begin{array}{c}87 \\
359 \\
83.7\end{array}$ & $\begin{array}{c}69 \\
250 \\
58.3\end{array}$ & $\begin{array}{c}37 \\
107 \\
25.0\end{array}$ & $\begin{array}{c}60 \\
175 \\
40.8\end{array}$ & $\begin{array}{c}71 \\
219 \\
51.0\end{array}$ & $\begin{array}{c}41 \\
106 \\
24.7\end{array}$ & $\begin{array}{l}44 \\
91 \\
21.2\end{array}$ \\
\hline Subject 8 & $\begin{array}{l}\% \mathrm{P} \\
\mathrm{B} / \mathrm{N}_{x} \\
\% \mathrm{C}\end{array}$ & $\begin{array}{c}85 \\
562 \\
64.0\end{array}$ & $\begin{array}{c}69 \\
284 \\
32.0\end{array}$ & $\begin{array}{c}63 \\
253 \\
29.0\end{array}$ & $\begin{array}{r}97 \\
872 \\
100\end{array}$ & $\begin{array}{r}99 \\
783 \\
90\end{array}$ & $\begin{array}{r}92 \\
516 \\
59\end{array}$ & $\begin{array}{r}78 \\
365 \\
42\end{array}$ & $\begin{array}{r}56 \\
263 \\
30\end{array}$ & $\begin{array}{r}93 \\
496 \\
57\end{array}$ & $\begin{array}{r}83 \\
363 \\
42\end{array}$ & $\begin{array}{r}49 \\
236 \\
27\end{array}$ \\
\hline Subject 9 & $\begin{array}{l}\% \mathrm{P} \\
\mathrm{B} / \mathrm{N}_{\mathrm{x}} \\
\% \mathrm{C}\end{array}$ & $\begin{array}{c}96 \\
625 \\
44.8\end{array}$ & $\begin{array}{c}79 \\
292 \\
20.9\end{array}$ & $\begin{array}{c}71 \\
213 \\
15.3\end{array}$ & $\begin{array}{l}96 \\
1,394 \\
100\end{array}$ & $\begin{array}{c}97 \\
872 \\
63.4\end{array}$ & $\begin{array}{c}90 \\
523 \\
37.5\end{array}$ & $\begin{array}{c}88 \\
462 \\
33.1\end{array}$ & $\begin{array}{c}92 \\
859 \\
61.6\end{array}$ & $\begin{array}{c}97 \\
1,074 \\
77.0\end{array}$ & $\begin{array}{l}100 \\
938 \\
67.3\end{array}$ & $\begin{array}{c}84 \\
424 \\
41.6\end{array}$ \\
\hline
\end{tabular}

* Symbols as in Table I.

TABLE V

Comparative influence of extreme concentrations of sodium, potassium, calcium, creatinine, citrate, and ammonium on phagocytosis of Staphylococci in lactated Ringer's solution*

\begin{tabular}{|c|c|c|c|c|c|c|c|c|c|}
\hline Altered solute & $\begin{array}{l}\text { Concen- } \\
\text { tration }\end{array}$ & $\% \mathrm{P}$ & $\mathrm{B} / \mathbf{N}_{\mathbf{x}}$ & $\% \mathrm{C}$ & $\mathrm{Na}$ & $\mathbf{K}$ & $\mathrm{Cl}$ & Lactate & $\mathrm{Ca}$ \\
\hline & $m M / L$ & & & & $m M / L$ & $m M / L$ & $m M / L$ & $m M / L$ & $m M / L$ \\
\hline $\begin{array}{l}\text { Sodium } \\
\text { Potassium } \\
\text { Calcium } \\
\text { Calcium } \\
\text { Creatinine } \\
\text { Citrate } \\
\text { Ammonium } \\
\text { Serum } \\
\text { Lactated Ringer's solution } †\end{array}$ & $\begin{array}{c}400 \\
130 \\
0 \\
15 \\
31.8 \\
15.8 \\
70\end{array}$ & $\begin{array}{r}16 \\
84 \\
98 \\
98 \\
94 \\
95 \\
77 \\
100 \\
100\end{array}$ & $\begin{array}{l}\quad 66 \\
1,260 \\
2,988_{-2} \\
3,963_{-12} \\
1,959_{-5} \\
1,688_{-3} \\
1,618_{-8} \\
3,377_{-24} \\
3,629^{2}\end{array}$ & $\begin{array}{r}1.8 \\
34.7 \\
82.3 \\
109.2 \\
54.0 \\
46.5 \\
44.6 \\
91.4 \\
100.0\end{array}$ & $\begin{array}{l}400 \\
130 \\
130 \\
130 \\
130 \\
130 \\
130\end{array}$ & $\begin{array}{r}4 \\
130 \\
4 \\
4 \\
4 \\
4 \\
4 \\
4 \\
4\end{array}$ & $\begin{array}{l}379 \\
235 \\
103 \\
136 \\
109 \\
109 \\
109 \\
109 \\
109\end{array}$ & $\begin{array}{l}28 \\
28 \\
28 \\
28 \\
28 \\
28 \\
28 \\
28 \\
28\end{array}$ & $\begin{array}{r}1.5 \\
1.5 \\
0 \\
15.0 \\
1.5 \\
1.5 \\
1.5 \\
1.5 \\
1.5\end{array}$ \\
\hline
\end{tabular}

* Symbols as in Table I.

† Control. 
DEPRESSION OF PHAGOCYTOSIS BY SOLUTE CONCENTRATIONS OF KIDNEY OR URINE

TABLE VI

Effect of $p H$ on phagocytosis of E. coli in plasma-leukocyte suspensions*

\begin{tabular}{lrrrrrr}
\hline \hline Mixtures & $\% \mathrm{P}$ & $\mathrm{B} / \mathrm{N}_{\mathbf{x}}$ & \multicolumn{1}{c}{$\% \mathrm{C}$} & $\mathrm{pH}+$ & $\mathrm{pH}$, & $0.06 \mathrm{~N} \mathrm{HCl}$ \\
\hline $1+$ & 94 & 622 & 138.0 & 7.3 & 7.2 & 0 \\
$2 \S$ & 86 & 462 & 103.0 & 7.5 & 6.9 & 0 \\
3 (control) & 95 & 450 & 100.0 & 7.6 & 7.0 & 0 \\
4 & 82 & 532 & 118.0 & 6.2 & 6.2 & .2 \\
5 & 3 & 8 & 1.8 & 5.4 & 5.5 & .3 \\
6 & 3 & 4 & .9 & 5.0 & 5.0 & .4 \\
\hline
\end{tabular}

* Symbols as in Table I; $0.8 \mathrm{ml}$ plasma-leukocyte suspension diluted with $4.0 \mathrm{ml}$ lactated Ringer's solution.

$\dagger \mathrm{pH}_{1}$ was determined when phagocytosis began and $\mathrm{pH}_{2}$ when it was completed.

Whole blood substituted for plasma-leukocyte suspension.

$\$ 0.4 \mathrm{ml}$ plasma-leukocyte suspension instead of $0.8 \mathrm{ml}$. .

be encountered in urine (Table V). The concentration of sodium, $400 \mathrm{mM}$ per $\mathrm{L}$, was close to the maximum observed in the renal medulla (1). The test solutions were examined simultaneously with leukocytes from the same sample of fresh human blood; hence, the results permit direct comparison of the different solutes. Although high concentrations of potassium, ammonium, creatinine, and citrate impaired phagocytosis of Staphylococci the inhibitory action of these solutes was mild compared to that of sodium. The extreme concentrations of calcium failed to influence phagocytosis significantly (see Table V).

E. $p H$. Phagocytosis was sharply reduced between $\mathrm{pH} 5.4$ and 6.2. The findings presented in Table VI were reproduced in three additional experiments. The neutrophiles at $\mathrm{pH} 5.4$ or less were shrunken and the nuclear lobes fused. Although the neutrophiles exhibited good phagocytosis at $\mathrm{pH} 6.2$, they seemed to be extruding cytoplasm and clumping in it. The E. coli were also clumped in the extruded cytoplasm.

TABLE VII

Effect of glucose on phagocytosis of Staphylococci and E. coli*

\begin{tabular}{|c|c|c|c|c|}
\hline Subject & Glucose & $\% \mathrm{P}$ & $\mathbf{B} / \mathbf{N}_{\mathbf{x}}$ & $\% \mathrm{C}$ \\
\hline & $\%$ & & & \\
\hline \multicolumn{5}{|c|}{$\begin{array}{l}\text { Phagocytosis of } \\
\text { Staphylococci }\end{array}$} \\
\hline 1 & $\begin{array}{l}12.5 \\
7.4 \\
4.8 \\
2.5 \\
1.25 \\
\dagger\end{array}$ & $\begin{array}{r}32 \\
90 \\
91 \\
99 \\
100 \\
100\end{array}$ & $\begin{array}{l}200 \\
1,220 \\
1,255 \\
1,594 \\
2,894_{-4} \\
3,304_{-2}\end{array}$ & $\begin{array}{r}6.1 \\
36.9 \\
38.0 \\
48.2 \\
87.6 \\
100\end{array}$ \\
\hline 2 & $\begin{array}{c}10.0 \\
2.0 \\
.05 \\
\dot{t}\end{array}$ & $\begin{array}{l}62 \\
75 \\
92 \\
90\end{array}$ & $\begin{array}{c}431 \\
871 \\
1,786_{-2} \\
1,984_{-1}\end{array}$ & $\begin{array}{l}21.7 \\
43.9 \\
90.0 \\
100\end{array}$ \\
\hline 3 & $\begin{array}{l}8.0 \\
6.0 \\
2.0 \\
.05 \\
\dagger\end{array}$ & $\begin{array}{l}73 \\
88 \\
97 \\
92 \\
97\end{array}$ & $\begin{array}{c}790 \\
992_{-1} \\
2,000 \\
2,113 \\
2,454_{-3}\end{array}$ & $\begin{array}{r}32 \\
42 \\
81 \\
86 \\
100\end{array}$ \\
\hline \multicolumn{5}{|c|}{$\begin{array}{l}\text { Phagocytosis of } \\
\text { E. coli }\end{array}$} \\
\hline 4 & $\begin{array}{r}10 \\
5 \\
2 \\
\dagger\end{array}$ & $\begin{array}{r}4 \\
98 \\
99 \\
98\end{array}$ & $\begin{array}{r}4 \\
497 \\
786 \\
749\end{array}$ & $\begin{array}{r}1 \\
66 \\
105 \\
100\end{array}$ \\
\hline
\end{tabular}

* Symbols as in Table I.

† Control. 
TABLE VIII

Phagocytosis of Staphylococc in normal urine*

\begin{tabular}{|c|c|c|c|c|c|c|c|c|c|c|}
\hline Subject & $\% \mathrm{P}$ & $\mathbf{B} / \mathbf{N}_{\mathbf{x}}$ & $\mathrm{B} / \mathrm{N}_{0}$ & $\% \mathrm{C}$ & mOsm & $\mathrm{Na}$ & Urea & $\mathbf{K}$ & $\mathrm{Cl}$ & $\mathrm{pH}$ \\
\hline & & & & & & $m M / L$ & $m M / L$ & $m M / L$ & $m M / L$ & \\
\hline $\begin{array}{l}1 \\
2 \\
3 \\
4\end{array}$ & $\begin{array}{r}54 \\
17 \\
40 \\
3\end{array}$ & $\begin{array}{c}509 \\
39 \\
644_{-1} \\
8\end{array}$ & $\begin{array}{l}2664 \\
3767 \\
2339 \\
2211\end{array}$ & $\begin{array}{r}19.0 \\
1.0 \\
27.5 \\
.4\end{array}$ & $\begin{array}{c}589 \\
>860 \dagger \\
\mathbf{4 8 4} \\
>\mathbf{8 6 0}\end{array}$ & $\begin{array}{l}155 \\
170 \\
147 \\
155\end{array}$ & $\begin{array}{l}179 \\
605 \\
124 \\
600\end{array}$ & $\begin{array}{l}64 \\
96 \\
49 \\
19\end{array}$ & $\begin{array}{l}196 \\
210 \\
183 \\
152\end{array}$ & $\begin{array}{l}7.1 \\
6.5\end{array}$ \\
\hline
\end{tabular}

* Symbols as in Table I.

$\uparrow$ Maximum value measurable on the osmometer.

F. Glucose. As shown in Table VII, inhibition of phagocytosis usually began above 2.0 per cent and became marked above 7 per cent.

\section{Phagocytosis in urine}

A. Normal and abnormal urines. The amount of phagocytosis occurring in urines ranged from 0 to 100 per cent of the controls as shown in Tables VIII, IX, and X. Phagocytosis was inhibited by urines with high osmolarity and high urea concentrations. In urines with osmolarity greater than $860 \mathrm{mOsm}$, or urea concentration greater than $400 \mathrm{mM}$ per L, the phagocytic index (\%C) never exceeded 8.6. The effects of the other factors studied are not clear. The infected urine of Subject 3 (see Table X), a diabetic with slight glycosuria, reduced phagocytosis to 1 per cent of control values, even though osmolarity and $\mathrm{pH}$ were not markedly abnormal. Depression of phagocytosis might be partly attributed to the low concentration of sodium. As shown in Table I, the concentration of $58 \mathrm{mM}$ per L sodium in this subject's urine reduced phagocytosis about 50 per cent when titrated in Ringer's solution. It is also possible that bacterial toxins injured the leukocytes in this case.
B. Effect of dilution or dialysis of urine on phagocytosis. Urines were obtained from subjects before and after they drank water in order to determine if the inhibitory effect of urine on phagocytosis can be removed by dilution in vivo. In addition, the nondialyzable portions of concentrated urines were examined for their inhibitory effect on phagocytosis.

The results in Table XI demonstrated that ingestion of water can abolish the inhibitory effect of concentrated urine on phagocytosis, and they support the concept that high osmolarity and high concentrations of urea inhibit phagocytosis in urine. Again there was marked inhibition of phagocytosis at osmolarity greater than $860 \mathrm{mOsm}$ and urea concentrations more than $400 \mathrm{mM}$ per L. Loss of the inhibitory effect by dialysis of concentrated urine can also be explained by a reduction in tonicity, and clearly demonstrates that the inhibitors to phagocytosis are absent from the nondialyzable fraction. A rise in $\mathrm{pH}$ might have contributed to the improved phagocytosis both in dialyzed urines and in those obtained after water diuresis, but this possibility was not supported by the observation that the $\mathrm{pH}$ of the concentrated urines did not fall below 5.8, a level that

TABLE IX

Phagocytosis of E. coli in normal urine*

\begin{tabular}{|c|c|c|c|c|c|c|c|c|c|c|}
\hline Subject $\dagger$ & $\% \mathbf{P}$ & $B / N_{x}$ & $B / N_{0}$ & $\% \mathrm{C}$ & mOsm & $\mathrm{Na}$ & Urea & $\mathbf{K}$ & $\mathrm{Cl}$ & $\mathrm{pH}$ \\
\hline & & & & & & $m M / L$ & $m M / L$ & $m M / L$ & $m M / L$ & \\
\hline $\begin{array}{l}1 \mathrm{a} \\
1 \mathrm{~b} \\
1 \mathrm{c} \\
1 \mathrm{~d} \\
2 \\
3 \mathrm{a} \\
3 \mathrm{~b}\end{array}$ & $\begin{array}{r}11 \\
14 \\
0 \\
16 \\
93 \\
2 \\
94\end{array}$ & $\begin{array}{r}31 \\
42 \\
0 \\
50 \\
501 \\
7 \\
540\end{array}$ & $\begin{array}{r}455 \\
455 \\
455 \\
455 \\
1,101_{-4} \\
1,181 \\
1,181\end{array}$ & $\begin{array}{r}6.8 \\
9.2 \\
0.0 \\
11.0 \\
45.5 \\
.6 \\
45.7\end{array}$ & $\begin{array}{r}>860 \\
540 \\
>860 \\
548 \\
292 \\
>860 \\
188\end{array}$ & $\begin{array}{r}156 \\
70 \\
95 \\
94 \\
49 \\
163 \\
37\end{array}$ & $\begin{array}{c}428 \\
340 \\
567 \\
283 \\
59.5 \\
388 \\
75\end{array}$ & $\begin{array}{l}115 \\
30 \\
74 \\
34 \\
12.4 \\
54.0 \\
11.0\end{array}$ & $\begin{array}{r}181 \\
43 \\
64 \\
83 \\
34.0 \\
128.0 \\
20.0\end{array}$ & $\begin{array}{l}6.5 \\
6.3 \\
6.0 \\
6.3 \\
7.0 \\
5.8 \\
7.1\end{array}$ \\
\hline
\end{tabular}

* Symbols as in Table I.

$\dagger$ Numbers refer to the human subject and letters to different samples from that subject. 
TABLE $\mathbf{X}$

Phagocytosis of Staphylococci in abnormal urine*

\begin{tabular}{|c|c|c|c|c|c|c|c|c|c|c|c|}
\hline Subject's diagnosis & $\% \mathrm{P}$ & $\mathrm{B} / \mathrm{N}_{\mathbf{x}}$ & $\mathrm{B} / \mathrm{N}_{\mathbf{0}}$ & $\% \mathrm{C}$ & mOsm & $\mathrm{Na}$ & Urea & $\mathbf{K}$ & $\mathrm{Cl}$ & $\mathrm{pH}$ & Glucose \\
\hline & & & & & \multicolumn{7}{|c|}{$m M / L m M / L m M / L m M / L$} \\
\hline $\begin{array}{l}\text { 1. Chronic pyelonephritis; } \\
\text { no bacteria or pyuria in } \\
\text { this urine }\end{array}$ & 26 & 87 & 1,118 & 8.5 & 759 & 214 & 263 & 70 & 202 & 7.4 & 0 \\
\hline $\begin{array}{l}\text { 2. Rt. staghorn calculus. } \\
K \text {. pneumoniae cultured } \\
\text { from urine }\end{array}$ & 26 & 146 & 1,003 & 14.5 & undetermined & 90 & 143 & 36 & 70 & 6.0 & 0 \\
\hline $\begin{array}{l}\text { 3. Diabetes; exacerbation } \\
\text { acute pyelonephritis; } P \text {. } \\
\text { mrabilis and } E \text {. coli in } \\
\text { pyuric urine }\end{array}$ & 8 & 24 & 2,221 & 1.0 & 350 & 58 & 188 & 22 & 55 & 6.5 & $<1$ \\
\hline $\begin{array}{l}\text { 4. Hyperthyroid; atonic } \\
\text { bladder; } P \text {. aeruginosa } \\
\text { cultured from pyuric urin }\end{array}$ & 100 & 440 & 1,474 & 30.0 & 375 & 83 & 178 & 39 & 89 & 6.3 & 0 \\
\hline
\end{tabular}

* Symbols as in Table I.

TABLE XI

Influence of water diuresis on the inhibitory effect of urine on phagocytosis of E. coli and Staphylococci*

\begin{tabular}{|c|c|c|c|c|c|c|c|c|c|c|}
\hline Urine & $\% \mathrm{P}$ & $\mathrm{B} / \mathrm{N}_{\mathbf{x}}$ & $\mathrm{B} / \mathrm{N}_{\mathrm{c}}$ & $\% \mathrm{C}$ & mOsm & $\mathrm{Na}$ & Urea & $\mathbf{K}$ & $\mathrm{Cl}$ & $\mathrm{pH}$ \\
\hline $\begin{array}{l}\text { Subject } 1 \text {, with } \\
E \text {. coli }\end{array}$ & & & & & & $m M / L$ & $m M / L$ & $m M / L$ & $m M / L$ & \\
\hline $\begin{array}{l}\text { 1st } \\
\text { 2nd } \\
\text { after } \\
\text { drinking } \\
\text { water }\end{array}$ & $\begin{array}{l}15 \\
58\end{array}$ & $\begin{array}{r}38 \\
265\end{array}$ & $\begin{array}{l}455 \\
455\end{array}$ & $\begin{array}{r}8.4 \\
58.2\end{array}$ & $\begin{array}{r}>860 \\
564\end{array}$ & $\begin{array}{l}151 \\
136\end{array}$ & $\begin{array}{l}467 \\
250\end{array}$ & $\begin{array}{l}5.9 \\
9.4\end{array}$ & $\begin{array}{l}104 \\
118\end{array}$ & $\begin{array}{l}6.3 \\
6.6\end{array}$ \\
\hline $\begin{array}{l}\text { 3rd } \\
\text { after } \\
\text { drinking } \\
\text { water }\end{array}$ & 78 & 508 & 455 & 111.6 & 305 & 75 & 125 & 3.2 & & 7.1 \\
\hline \multicolumn{11}{|l|}{$\begin{array}{l}\text { Subject } 2 \text {, with } \\
E \text {. coli }\end{array}$} \\
\hline $\begin{array}{l}\text { 1st } \\
\text { 2nd } \\
\text { after } \\
\text { drinking } \\
\text { water }\end{array}$ & $\begin{array}{l}11 \\
91\end{array}$ & $\begin{array}{r}19 \\
736\end{array}$ & $\begin{array}{l}514 \\
514\end{array}$ & $\begin{array}{r}3.7 \\
143.2\end{array}$ & $\begin{array}{r}>860 \\
340\end{array}$ & $\begin{array}{l}268 \\
124\end{array}$ & $\begin{array}{r}416 \\
83\end{array}$ & $\begin{array}{l}5.4 \\
3.5\end{array}$ & & $\begin{array}{l}6.0 \\
7.5\end{array}$ \\
\hline $\begin{array}{l}\text { 1st, non- } \\
\text { dialyzable } \\
\text { portion } \dagger\end{array}$ & 89 & 494 & 514 & 92.2 & 260 & & & & & 7.1 \\
\hline \multicolumn{11}{|l|}{$\begin{array}{l}\text { Subject 3, with } \\
\text { Staphylococci }\end{array}$} \\
\hline $\begin{array}{l}\text { 1st } \\
\text { 2nd } \\
\text { after } \\
\text { drinking } \\
\text { water }\end{array}$ & $\begin{array}{r}3 \\
98\end{array}$ & $\begin{array}{r}8 \\
1,676\end{array}$ & $\begin{array}{l}2,211 \\
2,211\end{array}$ & $\begin{array}{r}.4 \\
75.8\end{array}$ & $\begin{array}{r}>860 \\
696\end{array}$ & $\begin{array}{l}155 \\
129\end{array}$ & $\begin{array}{l}600 \\
300\end{array}$ & $\begin{array}{l}19 \\
20\end{array}$ & $\begin{array}{l}152 \\
189\end{array}$ & $\begin{array}{l}5.9 \\
5.8\end{array}$ \\
\hline $\begin{array}{l}\text { 1st, non- } \\
\text { dialyzable } \\
\text { portion } †\end{array}$ & 100 & 1,718 & 2,211 & 77.7 & 266 & & & & & \\
\hline
\end{tabular}

* Symbols as in Table I.

† Dialyzed against running distilled water for 3 hours and then against lactated Ringer's solution for 1 hour. 
was not markedly inhibitory, as shown in Table $\mathrm{XI}$.

\section{Statistical analysis}

Calculation of correlation coefficients by the formula $r=\Sigma x^{2} y^{2} /\left(x^{2} y^{2}\right)^{\frac{1}{2}}$ of Pearson and Lee disclosed a significant inverse relationship $(p=.01)$ between phagocytosis and concentrations of sodium, urea, and glucose, and between phagocytosis and osmolarity of normal urine.

\section{DISCUSSION}

Increasing concentrations of either sodium chloride, urea, or hydrogen ion progressively depressed phagocytosis until it was virtually abolished at a point well below the highest concentrations anticipated in the renal papilla. Moreover, sodium and urea in combination nearly abolished phagocytosis in concentrations that were only moderately inhibitory when these solutes were examined individually. This combined effect seemed to operate not only in artificial test solutions but also in those concentrated urines where phagocytosis was depressed to a greater extent than that anticipated from the effect of any solute alone. It is possible that the high concentration of these and other solutes in the kidney and urine also depressed phagocytosis in vivo in normal individuals. In diabetes, phagocytosis might be further depressed by severe glycosuria.

The mechanisms responsible for the antiphagocytic effect of these substances remain to be fully clarified, but good evidence suggests that high osmotic pressure is an important factor. A direct correlation could be made between the antiphagocytic properties of solutions and their osmolarities. The appearance of leukocytes also seemed to be related to tonicity. Thus in solutions made hypertonic with sodium, urea, and glucose, the leukocytes usually were contracted, while in hypotonic solutions they usually were swollen. The osmotic effectiveness of urea was also suggested by the observation that phagocytosis in $250 \mathrm{mM}$ per L sodium was sharply depressed by the addition of only $120 \mathrm{mM}$ per L urea, the amount required to produce an osmolarity shown to be critical for marked inhibition by sodium alone. The fact that urea has also been shown to be osmotically effective in preventing lysis of bacterial protoplasts in urine might indicate that this solute does not freely penetrate the cell water of microbial cells or leukocytes (2).

A possible application of these findings to the problem of urinary infection was suggested by the improved phagocytosis noted in urine obtained from subjects who had drunk water. The inhibitory effect of their hypertonic morning urines was abolished when their urines approached isotonicity. Occasional urines in the isotonic range inhibited phagocytosis, but these were heavily infected with bacteria whose toxins could have injured the leukocytes.

Inhibition of phagocytosis also occurred in hypotonic solutions, where the neutrophiles became swollen and distorted. Phagocytosis in markedly hypotonic urines was not examined because none was obtained for study, but it is reasonable to expect that excessive dilution of urine might injure leukocytes and reduce their activity.

Besides sodium and urea, hydrogen ion in high concentration also depressed phagocytosis sharply. This inhibitory effect occurred rather abruptly between $\mathrm{pH} 6.2$ and 5.5. Because the $\mathrm{pH}$ of urine in distal tubules and bladder may fall well below this level, acidity may be an important factor in preventing phagocytosis in the urinary tract. The fact that complement is rapidly destroyed at $\mathrm{pH}$ less than 5.5 (3) and is normally involved in phagocytosis suggests that loss of this substance may contribute to the inhibition of phagocytosis (4).

These studies have attempted to delineate some of the factors that might depress phagocytosis in the urinary system, but others remain to be explored. Observations summarized nearly 30 years ago in the review by Mudd, McCutcheon, and Lucke (5) indicate that many variables in the physical and chemical environment may stimulate or depress phagocytosis. It must be emphasized, also, that the present experiments were done in vitro and that only limited inferences may be drawn to conditions that prevail in vivo. Nonetheless, it is becoming increasingly evident that a variety of chemical conditions peculiar to the kidney might lower resistance to infection. Evidence has been obtained in other studies that the high urea concentration of the renal medulla favors infection by Proteus organisms because of their powerful urease (6), that intrarenal formation of 
ammonia inactivates the bactericidal system by destroying the fourth component of complement (7), and that hypertonicity allows protoplasts to develop and persist during antibiotic therapy (8). This evidence and that in the present study together may help explain why the kidney is uniquely susceptible to invasion by $E$. coli, Proteus, and other bacteria that possess little capacity to establish infection elsewhere.

\section{SUM M ARY}

In order to elucidate the peculiar susceptibility of the kidney to infection by bacteria that seldom produce disease elsewhere, a study was made of phagocytosis in fluids containing solutes in the variable concentrations found only in the kidney and urinary tract.

Within the range found in the normal kidney and urine, high concentrations of sodium, urea, and hydrogen ion inhibited phagocytosis by human leukocytes. Phagocytosis was inhibited by glucose in concentrations found in the urinary tract in diabetes mellitus. Highly concentrated human urines also depressed phagocytosis, but more dilute urines usually did not. Evidence has thus been obtained that phagocytosis might be impaired in the urinary system and that resistance to infection in the kidney might be thereby reduced.

\section{REFERENCES}

1. Lamdin, E. Mechanisms of urinary concentration and dilution, A.M.A. Arch. intern. Med. 1959, 103, 644. 103, 644.

2. Braude, A. I., and Siemienski, J. Urinary Protoplasts. To be published.

3. Boyd, William C. Fundamentals of Immunology, 3rd ed. New York, Interscience, 1956, p. 373.

4. Berry, L. J., and Spies, T. D. Phagocytosis. Medicine 1949, 28, 239.

5. Mudd, S., McCutcheon, M., and Lucké, B. Phagocytosis. Physiol. Rev. 1934, 14, 210.

6. Braude, A. I., and Siemienski, J. Role of bacterial urease in experimental pyelonephritis. J. Bact. 1960, 80, 171.

7. Beeson, P. B., and Rowley, D. The anticomplementary effect of kidney tissue. Its association with ammonia production. J. exp. Med. 1959, 110, 685.

8. Braude, A. I., Siemienski, J., and Jacobs, I. Protoplast formation in human urine. Trans. Ass. Amer. Phycns 1961, 74, 234. 